\title{
PAIRWISE RELATIVELY PRIME SOLUTIONS OF LINEAR DIOPHANTINE EQUATIONS
}

\author{
R. T. WORLEY \\ (Received 20 September 1982, revised 21 October 1982) \\ Communicated by J. H. Loxton
}

\begin{abstract}
It is shown that if $a_{1}, \ldots, a_{m}$ are relatively prime integers then for every integer $n$ the equation

$$
a_{1} x_{1}+a_{2} x_{2}+\cdots+a_{m} x_{m}=n
$$

has infinitely many solutions in pairwise relatively prime integers $x_{1}, \ldots, x_{m}$.

1980 Mathematics subject classification (Amer. Math. Soc.): 10 B 05.

In a recent paper [4] it was shown by an elementary method (a simple sieve using the Moebius function) that if the greatest common divisor $(a, b)=1$ then the diophantine equation

$$
a x+b y=n
$$

has solutions with $(x, y)=1$ and $x>y>0$ provided $n$ is sufficiently large. With little modification the proof shows that for all $n,(1)$ has solutions with $(x, y)=1$. More recently, B. H. Neumann asked for an elementary proof that if $(a, b, c)$ $=1$ then for all integers $n$

$$
a x+b y+c z=n
$$

has solutions with $(x, y)=(x, z)=(y, z)=1$, being dissatisfied with the fact that his proof used the infinitude of primes in arithmetical progressions. Since the result appears simple, it would be expected to be in the literature. However I have been unable to find the result mentioned in the obvious places (Dickson [1], LeVeque [3]). It seems that the need for relatively prime solutions has not arisen before.
\end{abstract}

(C) 1984 Australian Mathematical Society 0263-6115/84 \$A2.00+0.00 
In this note we show by elementary methods:

THEOREM. If $\left(a_{1}, a_{2}, \ldots, a_{m}\right)=1$ then for all integers $n$ the equations

$$
\begin{aligned}
& a_{1} x_{1}+a_{2} x_{2}+\cdots+a_{m} x_{m}=n, \\
& \left(x_{i}, x_{j}\right)=1, \quad 1 \leqslant i<j \leqslant m,
\end{aligned}
$$

have infinitely many solutions.

This result includes Neumann's case as $m=3$. The proof is by induction on $m$, the difficult case being a stronger version of the case $m=2$.

Lemma 1. If $\left(a_{1}, a_{2}\right)=1, z$ is odd and $(z, n)=1$ then for all integers $n$ the equations

$$
\begin{gathered}
a_{1} x_{1}+a_{2} x_{2}=n, \\
\left(x_{1}, x_{2}\right)=\left(x_{1}, z\right)=\left(x_{2}, z\right)=1
\end{gathered}
$$

have infinitely many solutions.

Proof. Since $\left(a_{1}, a_{2}\right)=1$ there is a solution $x_{1}=u_{0}, x_{2}=v_{0}$ of (3a). This generates a family

$$
x_{1}=u_{l}=u_{0}+l a_{2}, \quad x_{2}=v_{l}=v_{0}-l a_{1}, \quad l \in \mathbf{Z},
$$

of solutions of (3a). The aim of the proof is to show that if $r$ is sufficiently large

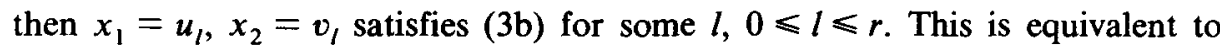
showing that $N_{r}$ is arbitrarily large for sufficiently large $r$, where

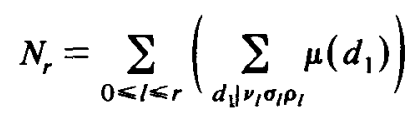

with $\nu_{l}=\left(u_{l}, v_{l}\right), \sigma_{l}=\left(u_{l}, z\right), \rho_{l}=\left(v_{l}, z\right)$ and $\mu$ denoting the Moebius function.

Since $x_{1}=u_{l}, x_{2}=v_{l}$ satisfy $(3 a)$, and $(z, n)=1$, it is clear that $\nu_{l}, \sigma_{l}$ and $\rho_{l}$ are pairwise relatively prime, so any divisor $d_{1}$ of $\nu_{l} \sigma_{l} \rho_{l}$ can be written uniquely as $d_{1}=\operatorname{def}$ where $d\left|\nu_{l}, e\right| \sigma_{l}$ and $f \mid \rho_{l}$. Thus

$$
N_{r}=\sum_{0 \leqslant l \leqslant r} \sum_{d \mid \nu_{l}} \mu(d) \sum_{e \mid \sigma_{l}} \mu(e) \sum_{f \mid \rho_{l}} \mu(f)
$$

which we rearrange as

$$
N_{r}=\sum_{d \mid n} \mu(d)\left\{\sum_{\substack{0 \leq|<r \\ d| \nu_{l}}} \sum_{e \mid \sigma_{l}} \mu(e) \sum_{f \mid \rho_{l}} \mu(f)\right\}
$$


since any divisor of $\nu_{l}$ must divide $n$. To continue with the proof we need the following result.

LEMMA 2. With the above notation, the $l$ for which $d \mid \nu_{l}$ form precisely one congruence class mod $d$, when $d \mid n$.

Proof. Plainly if $d \mid \nu_{l_{1}}$ then $d \mid \nu_{l}$ for all $l \equiv l_{1} \bmod d$. Conversely $d\left|\nu_{l_{1}}, d\right| \nu_{l_{2}}$ implies $d$ divides $u_{l_{1}}-u_{l_{2}}=a_{2}\left(l_{1}-l_{2}\right)$ and $d$ divides $a_{1}\left(l_{1}-l_{2}\right)$. Since $\left(a_{1}, a_{2}\right)$ $=1$ it follows that $l_{1} \equiv l_{2} \bmod d$. It just remains to show that $d \mid v_{1}$ for some $l$.

Consider the equation $a_{1} u_{k}+a_{2} v_{k}=n$, that is,

$$
a_{1}\left(u_{0}+a_{2} k\right)+a_{2}\left(v_{0}-a_{1} k\right)=n
$$

and let $d \mid n$. Set $\delta=\left(a_{2}, d\right)$ : then $\left(\delta, a_{1}\right)=1, \delta \mid a_{2}$ and $\delta|d| n=a_{1} u_{0}+a_{2} v_{0}$. Hence $\delta \mid u_{0}$, from which it follows that there exists an integer $k$ with $a_{2} k \equiv$ $-u_{0} \bmod d$. In other words, $a_{1} u_{k}+a_{2} v_{k}=n$ with $d \mid u_{k}$. Let $d^{*}=d \delta^{-1}$. Then $u_{k+t d^{*}}=u_{k}+a_{2} t d^{*}=u_{k}+a_{2}^{*} t d$ where $a_{2}^{*}=a_{2} \delta^{-1}$, so $d \mid u_{k+t d^{*}}$ for all integers $t$. On the other hand, $d \mid a_{2} v_{k}=n-a_{1} u_{k}$ means $d^{*} \mid v_{k}$. Since $\left(a_{1}, \delta\right)=1$ there is an integer $t$ such that

$$
a_{1} t \equiv v_{k} / d^{*} \bmod \delta
$$

Then $d=d^{*} \delta$ divides $v_{k}-a_{1} t d^{*}=v_{k+t d^{*}}$, and so $d \mid \nu_{l}$ for $l=k+t d^{*}$.

Proof of Lemma 1 (continued). We re-write the inner sum of (4) as

$$
\sum_{\substack{0 \leq l \leq r \\ l \equiv l_{1} \bmod d}} \sum_{e \mid \sigma_{l}} \mu(e) \sum_{f \mid \rho_{l}} \mu(f)
$$

and rearrange it as

$$
\sum_{e \mid z} \mu(e)\left\{\sum_{\substack{0 \leqslant l \leqslant r \\ l \equiv l_{1} \bmod d \\ e \mid u_{l}}} \sum_{f \mid \rho_{l}} \mu(f)\right\}
$$

where $l_{1}$ has the property that $d \mid \nu_{l_{1}}$. If $e \mid z$ has the property that $\left(e, a_{2}\right) \neq 1$, choose a prime $p \mid\left(e, a_{2}\right)$. Then $p|e| u_{l}, p \mid a_{2}, a_{1} u_{l}+a_{2} v_{l}=n$ would mean $p \mid(z, n)=1$, a contradiction. Hence if $\left(e, a_{2}\right) \neq 1$ then the inner sum in (6) is empty as there will be no $l$ for which $e \mid u_{l}$. We can therefore write the sum in (6) as

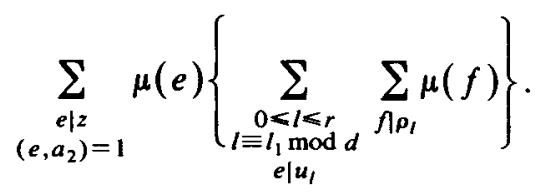


For $l \equiv l_{1} \bmod d$, we write

$$
u_{l}=u_{i_{1}}+k d a_{2}, \quad k \in \mathbf{Z} .
$$

Since $(d, e) \mid(n, z)=1$ and $\left(e, a_{2}\right)=1$ it is clear that $e \mid u_{l}$ for $k$ lying in a unique congruence class mod $e$; that is, $l$ lying in a unique congruence class mod $d e$. We can therefore write the inner sum in (7) as

$$
\sum_{\substack{0 \leqslant l \leqslant r \\ l \equiv l_{2} \bmod d e}} \sum_{f \mid \rho_{l}} \mu(f)
$$

where $e \mid \sigma_{l_{2}}$ and $d \mid \nu_{l_{2}}$.

We rearrange this sum as

$$
\sum_{f \mid z} \mu(f)\left\{\sum_{\substack{0 \leq 1 \leq r \\ l \equiv I_{2} \bmod d e \\ f \mid v_{\ell}}} 1\right\} .
$$

If $f \mid z$ has the property that $\left(f, a_{1}\right) \neq 1$, choose a prime $p \mid\left(f, a_{1}\right)$. Then $p|f| v_{l}$, $p \mid a_{1}, a_{1} u_{l}+a_{2} v_{l}=n$ would mean that $p \mid(2, n)=1$, which is impossible. Thus if $f \mid z$ the inner sum in (8) is empty unless $\left(f, a_{1}\right)=1$, so we can write (8) as

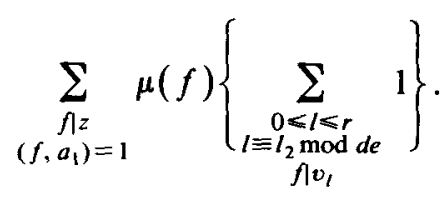

For $l \equiv l_{2} \bmod d e$ we write

$$
v_{l}=v_{l_{2}}-k \text { dea }_{1}, \quad k \in \mathbf{Z},
$$

If $f \mid z,\left(f, a_{1}\right)=1$ and $(f, e) \neq 1$ then $f$ cannot divide $v_{l}$ for any $l$, as $\left(\rho_{l}, \sigma_{l}\right)=1$. However if $(f, e)=1$ then $(d e, f)=1$ and $f \mid v$, for $k$ lying in a unique congruence class mod $f$. We can therefore write (9) as

$$
\sum_{\substack{\left.f \mid z \\ f, a_{1} e\right)=1}} \sum_{\substack{0 \leq 1<r \\ l \equiv l_{3} \bmod d e f}} 1=\sum_{\substack{f \mid z \\\left(f, a_{1} e\right)=1}} \mu(f)\left(\frac{r}{d e f}+O(1)\right) .
$$

Let $z_{e}$ denote the greatest divisor of $z$ prime to $e$. The above expression becomes

$$
\frac{r}{d e} \sum_{f \mid z_{a_{1} e}} \frac{\mu(f)}{f}+\mathrm{O}\left(\tau\left(z_{a_{1}}\right)\right)=\frac{r}{d e} \frac{\phi\left(z_{a_{1} e}\right)}{z_{a_{1} e}}+\mathrm{O}(\tau(z))
$$


where $\tau(n)$ denotes the number of divisors of $n$ and $\phi$ is the Euler function. Substituting in (7) we obtain the estimate

$$
\sum_{e \mid z_{a_{2}}} \frac{r}{d} \frac{\mu(e)}{e} \frac{\phi\left(z_{a_{1} e}\right)}{z_{a_{1} e}}+\mathrm{O}\left(\tau(z) \sum_{e \mid z_{a_{2}}} \mu(e)\right)=\frac{r}{d} \frac{\phi\left(z^{+}\right)}{z^{+}} \frac{\phi^{*}\left(z^{-}\right)}{z^{-}}+\mathrm{O}\left(\tau^{2}(z)\right)
$$

where $z^{+}$denotes the product of primes dividing one but not both $z_{a_{1}}$ and $z_{a_{2}}, z^{-}$ denotes the product of primes dividing both $z_{a_{1}}$ and $z_{a_{2}}$, and $\phi^{*}(z) / z=$ $\Pi_{p \mid z}\left(1-2 p^{-1}\right)$.

Finally we obtain the estimate

$$
N_{r}=r \frac{\phi(n)}{n} \frac{\phi\left(z^{+}\right)}{z^{+}} \frac{\phi^{*}\left(z^{-}\right)}{z^{-}}+O\left(\tau^{2}(z) \tau(n)\right)
$$

which is arbitrarily large for sufficiently large $r$.

RemarK 1. Note that $\phi^{*}\left(z^{-}\right) / z^{-}$is zero if $2 \mid z^{-}$. This occurs only when $z$ is even and both $a_{1}, a_{2}$ are odd, so the conclusion of Lemma 1 is valid when $z$ is even, provided $a_{1} a_{2}$ is even.

Remark 2. J. Loxton has observed that Lemma 1 could be proved using congruences and the Chinese Remainder Theorem. The above proof has the advantage of giving the estimate for $N_{r}$, and covering the case $z$ even, $a_{1} a_{2}$ even.

Proof of the theorem. The case $m=1$ is vacuous (or trivial, depending on your viewpoint). For $m \geqslant 2$ it is convenient to prove a slightly stronger result, namely.

Proposition. If $\left(a_{1}, \ldots, a_{m}\right)=1$, and $a_{1}, \ldots, a_{m}$ are ordered so that if $i \leqslant j$ then $a_{j}$ is not divisible by a higher power of 2 than $a_{i}$, then, for all integers $n$, the equations (2a), (2b) have infinitely many solutions in which $x_{1}, \ldots, x_{m-1}$ are odd.

Proof. The proof is by induction on $m$. For $m=2$ we consider cases, noting that $a_{2}$ must be odd.

(i) If $n$ is even, apply Lemma 1 with $z=1 . x_{1}$ must be odd for if $x_{1}$ were even then $x_{2}$ would have to be odd, so $a_{1} x_{1}=n-a_{2} x_{2}$ is odd, an impossiblity.

(ii) If $n$ is odd and $a_{1}$ is even, apply Lemma 1 with $z=2$ (note Remark 1).

(iii) If $n, a_{1}$ and $a_{2}$ are all odd then $\left(a_{1}, 2 a_{2}\right)=1$ so by Lemma 1 with $z=1$ there exist $x_{1}, x_{2}$ satisfying $a_{1} x_{1}+2 a_{2} x_{2}=n,\left(x_{1}, x_{2}\right)=1$. In this case $x_{1}$ is plainly odd, and $x_{1}^{\prime}=x_{1}, x_{2}^{\prime}=2 x_{2}$ satisfy $a_{1} x_{1}^{\prime}+a_{2} x_{2}^{\prime}=n,\left(x_{1}^{\prime}, x_{2}^{\prime}\right)=1$. This proves the proposition when $m=2$. 
Now suppose the proposition is true for $m-1$. Let $g=\left(a_{1}, a_{m}\right)$, which is odd and satisfies $\left(g, a_{2}, \ldots, a_{m-1}\right)=1$. By the inductive assumption there exist solutions $x_{2}, \ldots, x_{m-1}, q$ of

$$
\begin{gathered}
a_{2} x_{2}+\cdots+a_{m-1} x_{m-1}+g q=n, \\
\left(x_{2} \cdots x_{m-1}, q\right)=1, \quad\left(x_{i}, x_{j}\right)=1, \quad 2 \leqslant i<j \leqslant m-1, \\
x_{2} \cdots x_{m-1} \text { odd. }
\end{gathered}
$$

Now (2a) is satisfied by any solution of $a_{1} x_{1}+a_{m} x_{m}=g q$, that is, $a_{1}^{\prime} x_{1}+a_{m}^{\prime} x_{m}$ $=q$ where $a_{1}^{\prime}=a_{1} / g, a_{m}^{\prime}=a_{m} / g$. By Lemma 1 , since $z=x_{2} \cdots x_{m-1}$ is odd and prime to $q$, there are infinitely many solutions of this satisfying $x_{1}$ odd and $\left(x_{1}, x_{m}\right)=\left(x_{1}, x_{2} \cdots x_{m-1}\right)=\left(x_{m}, x_{2} \cdots x_{m-1}\right)=1$. Thus $x_{1}, \ldots, x_{m}$ satisfy (2a), (2b) and $x_{1}, \ldots, x_{m-1}$ are odd. This completes the proof by induction.

RemarK 3. For any odd integer $z$ with $(z, n)=1$ the above proof can easily be modified to ensure the solutions $x_{1}, \ldots, x_{m}$ are also prime to $z$. If $z$ is even and $(z, n)=1$, let $z=2^{r} z_{1}$ with $z_{1}$ odd. The solutions $x_{1}, \ldots, x_{m}$ prime to $z_{1}$ also have $x_{1}, \ldots, x_{m-1}$ odd, so they are prime to $z$. Plainly $x_{m}$ can (and will) be odd, and therefore prime to $z$, if $a_{1}+\cdots+a_{m-1}$ is even.

\section{References}

[1] L. E. Dickson, History of the theory of numbers (Chelsea, 1952).

[2] B. H. Neumann, Some finite groups with few defining relations, ANU Mathematics Research Report 40 (Australian National University, 1982).

[3] W. J. LeVeque, Reviews in number theory (Amer. Math. Soc., Providence, R.I., 1974).

[4] R. T. Worley, 'Denominator sequences for continued fractions III', J. Austral. Math. Soc. Ser. A 26 (1978), 53-56.

Department of Mathematics

Monash University

Clayton, Victoria 3168

Australia 